\title{
Notes de la Rédaction
}

Cette livraison contient des articles et des comptes rendus qui soulèvent plusieurs questions sur la mesure dans laquelle il convient de généraliser en se basant sur des échantillons de grandeurs limitées. Ce sujet touche de près les lecteurs et les auteurs en tant que consommateurs et créateurs d'ouvrages érudits.

Moore et Pfeiffer dans une étude de recherches sur la condition physique des fermiers âgés dans l'industrie laitière mentionnent qu'ils ont été désappointés par le faible taux de participation produisant un petit groupe témoin. Ils invitent donc les lecteurs à interpréter prudemment les résultats puisque l'échantillon est modique et qu'il n'a pas été prélevé au hasard. Ils énumèrent les raisons pour lesquelles seulement 36 des 517 fermiers faisant partie de l'étude originale ont répondu à l'enquête. Certains par exemple ont été classifiés "refus" ou inéligibles parce qu'ils n'étaient pas âgés de 50 ans et plus, tandis que d'autres n'ont jamais été contactés. Le rédacteuren-chef, section des sciences de la santé, et moi-même sommes d'avis que cette étude mérite néanmoins d'être publiée puisque les auteurs nous mettent en garde contre le danger de généraliser. En dépit de ces risques, les résultats contribuent au progrès de la science et peuvent certainement inspirer des études de recherches plus poussées.

L'article sur la fratrie rédigé par Gold se distingue en tant qu'étude de recherches théoriquement innovatrice basée sur un petit échantillon spécialisé. Les personnes possédant certaines particularités distinctives (ni frères ni soeurs vivants, jumeaux, jamais mariés ou sans enfants) ont été exclus dans le but de contrôler la variabilité découlant de ces facteurs. Gold a choisi ses sujets dans des endroits fréquentés par les gens âgés désireux de s'impliquer à la société et plus en mesure de participer. Elle prévient le lecteur qu'il y a possibilité de biais et elle offre des données illustrant le degré de représentativité de son groupe témoin. Tel que noté par l'auteur, cette stratégie convient aux études de recherches exploratoires ou à la génération de théorie.

Dans son article sur le taux de réponses et de réussites, Marshall recommande l'adoption d'une terminologie standard pour décrire les procédures d'échantillonnage dans les enquêtes de recherches et retrace les procédés utilisés dans trois études de recherches canadiennes. Le quatrième article, rédigé par Rosenthal, est basé sur une de ces enquêtes. Le texte et la première note en bas de page illustrent précisément la façon dont les données d'échantillonnage devraient être publiées dans les revues.

La section des comptes rendus souligne également l'importance de présenter exactement les données d'échantillon. A l'issue d'une analyse d'un important rapport d'une enquête de l'Association des infirmières et infirmiers auxiliaires diplômés de l'Ontario, French remarque que le pourcentage de réponses à un sondage était d'un piètre $29 \%$ et que l'interprétation 
des résultats a été obstrué car les auteurs ont négligé de mentionner le nombre d'institutions représentées dans l'échantillon ou le nombre d'individus dans chaque institution.

Idler offre un compte rendu sur un livre écrit par Wan basé sur un échantillon important national américain utilisé fréquemment par les gérontologues sociaux dans leurs travaux d'analyses. Puisque l'étude est basée sur un échantillon de "chefs de famille", elle ne représente pas les femmes mariées. Idler signale qu'un bon nombre des généralisations contenues dans le livre sont par conséquent imparfaites et, de plus, il mentionne que l'auteur ne souligne pas suffisamment les implications dérivant de l'emploi d'échantillons limités dans l'analyse.

Je suis d'avis que nous devrions tolérer les petits échantillons nonsystématiques. De telles études, basées sur des échantillons modiques ou faiblement représentatifs ont après tout produit parmi les ouvrages les plus illustres dans le domaine de la gérontologie. On n'a qu'à se reporter à l'étude longitudinale de Duke qui nous a fourni une profusion de données. Au tout début en 1955, elle contenait 260 participants et à l'issue de la deuxième phase en 1961, son nombre s'est vu réduit à 183 . Du reste, il s'agissait essentiellement d'un échantillon déterminé par le quota et les bénévoles désirés ont été classifiés selon leur âge, sexe, race et condition socioéconomique dans le but de refléter les traits distinctifs de la population des trois comtés enquêtés en Caroline du Nord. Le panel d'origine, tel que reconnu par les investigateurs, favorisait une condition socio-économique plus élevée, un niveau de fonctionnement plus soutenu et une meilleure santé (Busse, Maddox and Associates, 1985, pp. 18-20). Les investigateurs ont néanmoins développé et mis à l'épreuve certaines théories comme par exemple la théorie d'action du vieillissement (même si ces caractéristiques d'échantillonnage sont des variables importantes dans cette théorie) mais ils se sont bien gardés de généraliser à une plus grande population.

Certains travaux sont basés sur un groupe composé tellement accidentellement qu'il ne convient absolument pas de le qualifier de groupe témoin. Néanmoins, ces études peuvent être extrêmement enrichissantes. Une description précise et détaillée sur la marche à suivre employée pour prélever l'échantillon est de rigueur et permet au lecteur d'évaluer la fiabilité des résultats et la mesure dans laquelle ils peuvent être généralisés. Cependant, le chercheur n'a pas toujours l'intention de généraliser, désirant plutôt explorer les tendances causales qui relient les variables ou cherchant à développer des concepts positifs pour comprendre le phénomène. Dans une analyse causale, s'il y a variabilité dans l'échantillon sur les variables analysées, il est alors possible de mettre la théorie à l'épreuve.

L'investigateur doit définir clairement ce qu'il désire obtenir de son échantillon et le lecteur doit avoir une idée précise de ce qu'il pourra déduire des échantillons qui sont à la base des études de recherches. Une description précise et détaillée non seulement de l'échantillon mais des méthodes employées pour l'obtenir sont essentiels pour répondre aux objectifs établis. 